Pacific

Journal of

Mathematics

\title{
A CONE SPLITTING THEOREM FOR ALEXANDROV SPACES
}

Stephanie B. Alexander AND Richard L. BishoP 


\title{
A CONE SPLITTING THEOREM FOR ALEXANDROV SPACES
}

\author{
StePhanie B. AleXANDER AND RichaRd L. BishoP
}

\begin{abstract}
By a cone is meant a warped product $I \times{ }_{g} F$, where $I$ is an interval and the warping function $g: I \rightarrow \mathbb{R}_{\geq 0}$ lies in $\mathscr{F} K$, i.e., satisfies $g^{\prime \prime}+K g=0$. Cones include metric products and linear cones $(K=0)$, hyperbolic, parabolic, and elliptical cones $(K<0)$, and spherical suspensions $(K>0)$. A cone over a geodesic metric space supports a natural $K$-affine function, that is, a function whose restriction to every unit-speed geodesic is in $\mathscr{F} K$. Conversely, the main theorems of this paper show that on an Alexandrov space $X$ of curvature bounded below or above, the existence of a nonconstant $K$-affine function $f$ forces $X$ to split as a cone (subject to a boundary condition or geodesic completeness, respectively).

For $K=0$ and curvature bounded below, $X$ splits as a metric product with a line; this case is due to Mashiko (2002). Some special cases for complete Riemannian manifolds were discovered much earlier: by Obata (1962), for $K>0$, with the strong conclusion that $X$ is a standard sphere; and by Innami (1982), for $K=0$. For $K<0$, with the additional assumption that $f$ has a critical point, our theorem now gives the dual to Obata's theorem, namely, $X$ is hyperbolic space.
\end{abstract}

\section{Introduction}

Cone constructions are intimately related to the study of spaces with curvature bounds. These fundamental constructions allow control of curvature bounds either above or below in the sense of Alexandrov (see definitions in Section 4), and appear as model spaces in rigidity theorems. The simplest cone construction is Cartesian product with a line. By Toponogov's Splitting Theorem [1959], a nonnegatively curved, complete Riemannian space that contains a line splits as a Cartesian product; the extension to Alexandrov spaces is due to Milka [1967]. By the Flat Strip Theorem, in a nonpositively curved Alexandrov space the convex hull of a collection of asymptotic lines splits as a Cartesian product (see [Bridson and Haefliger 1999]). Other cone constructions were introduced by Berestovskii in the

MSC2000: 53C20.

Keywords: Alexandrov spaces, warped products, affine functions.

The research described in this paper was made possible in part by Award No. RM1-2381-ST-02 of the U.S. Civilian Research \& Development Foundation for the Independent States of the Former Soviet Union (CRDF). 
context of curvature bounded above [Berestovskii 1983; Aleksandrov et al. 1986]. (See [Bridson and Haefliger 1999, Ch. II.3].) In the context of curvature bounded below, cone constructions were discussed by Burago, Gromov and Perelman in [Burago et al. 1992, §4], namely, linear, hyperbolic, parabolic and elliptic cones and spherical suspensions. These constructions can be traced to their classical roots by noting that $E^{n}$ is the linear cone over $S^{n-1} ; S^{n}$ is the spherical suspension of $S^{n-1}$; and $H^{n}$ is the hyperbolic cone over $H^{n-1}$, the parabolic cone over $E^{n-1}$, and the elliptic cone over $S^{n-1}$.

Here we introduce the notion of $K$-affine functions on geodesic metric spaces, and prove that in the presence of a curvature bound, such functions correspond precisely to the cone constructions. Our cone splitting theorem holds both for Alexandrov spaces of curvature bounded below (CBB), subject to a necessary boundary condition, and for geodesically complete Alexandrov spaces of curvature bounded above (CBA). In the latter setting, not only do the above-mentioned cones appear, but also spaces formed by gluing them together on their vertices.

For Alexandrov spaces of $\mathrm{CBB}$, the case $K=0$ (that is, the Cartesian product case) of our theorem is due to Mashiko [2002]. A new argument is needed to embed Mashiko's theorem in a family of splitting theorems, which moreover has a dual family for spaces of CBA.

Alexandrov spaces play a key role in the convergence theory of spaces with curvature bounds, and our cone splitting theorems should be a tool to identify limit spaces. Our theorems exhibit a new rigidity phenomenon: namely, if $X$ carries a nonconstant $K$-affine function but does not have $K$ as a curvature bound, then $X$ is a Cartesian product or a hyperbolic cone.

\section{Main theorems}

Let $\mathscr{F} K$ denote the family of (one-variable) solutions of the differential equation $f^{\prime \prime}+K f=0$. Then $\left(f^{\prime}\right)^{2}+K f^{2}$ is constant for $f \in \mathscr{F} K$. By definition, a $K$-affine function is a continuous function whose restriction to every unit-speed geodesic lies in $\mathscr{F} K$. We single out a special basis of $\mathscr{F} K: \mathrm{cs}_{K}$ and $\mathrm{sn}_{K}$ are the functions in $F_{F} K$ such that the value and derivative at 0 are 1,0 and 0,1 , respectively.

By a cone we mean a warped product $I \times{ }_{g} F$, where $I$ is an interval and the warping function $g: I \rightarrow \mathbb{R}_{\geq 0}$ is in $\mathscr{F} K$. For $K=0$, we obtain Cartesian products and linear cones, defined by $g=B$ and $g=B x$, respectively; for $K>0$, spherical suspensions, with $g=B \sin \sqrt{K} x$; and for $K<0$, hyperbolic, parabolic and elliptic cones, with $g=B \cosh \sqrt{-K} x, B \exp \sqrt{-K} x$, and $B \sinh \sqrt{-K} x$, respectively. (These are precisely the cones described somewhat differently in [Burago et al. 1992, §4].)

Cones over geodesic metric spaces support $K$-affine functions (Theorem 6.1). Moreover, on an Alexandrov space $X$ of CBB or CBA, the existence of a nonconstant $K$-affine function, subject to a boundary condition if the boundary is 
nonempty for $\mathrm{CBB}$, and geodesic completeness for $\mathrm{CBA}$, forces the space to split as a cone (Theorems 3.1 and 3.2). The following two theorems summarize the correspondence between $K$-affine functions and cone structures. Linear cones are the only type not occurring on the following lists; this is because the natural affine functions associated with them are constant functions, which have no structural implications.

Theorem 2.1. Let $X$ be a complete, finite-dimensional Alexandrov space of CBB by $\bar{K}$. Then $X$ carries a nonconstant $K$-affine function $f$ that satisfies the boundary condition ( $\dagger$ ) below if and only if $\bar{K} \leq K$ and $X$ splits as a cone over a complete, finite-dimensional Alexandrov space $F$, in one of the following ways:

(1) $K>0$ and $X=[0, \pi / \sqrt{K}] \times_{\mathrm{sn}_{K} x} F$, a spherical suspension of an Alexandrov space $F$ of $C B B$ by 1 .

(2) $K<0$ and $X=[0, \infty) \times_{\operatorname{sn}_{K} x} F$, an elliptic cone over an Alexandrov space $F$ of $C B B$ by 1.

(3) $K<0$ and $X=\mathbb{R} \times \exp \sqrt{-K} x$, a parabolic cone over an Alexandrov space $F$ of $C B B$ by 0 .

(4) $K<0$ and $X=\mathbb{R} \times \times_{\cosh \sqrt{-K} x} F$, a hyperbolic cone over an Alexandrov spaces $F$ of $C B B$ by $\bar{K}$.

(5) $K=0$ and $X=\mathbb{R} \times F$, a Cartesian product where $F$ is an Alexandrov space of $C B B$ by $\bar{K}$.

In cases (1), (2) and (3), $X$ has CBB by $K$.

If $\partial X \neq \varnothing$, the boundary condition on $f$ in Theorem 2.1 is:

The tautological extension of $f$ to the double of $X$ is $K$-affine.

Case (5) of Theorem 2.1 was proved by Mashiko [2002], who used different regularity hypotheses on $f$. Here we merely assume $(\dagger)$ at boundary points, while in Mashiko's article, $f$ is assumed of $D^{2,2}$ class. This means that

(a) the differential $D f_{p}$ of $f$ is well defined and continuous on the direction space $\Sigma_{p}$ (= unit tangent vectors) at every $p \in X$ (" $f \in D^{1}$ "), and similarly for the differential of $D f_{p}$ on the direction space of $\Sigma_{p}$ at every $u \in \Sigma_{p}$ (" $f \in D^{2}$ "); and

(b) the average directional derivative of $f$ at any $p \in X$ vanishes, and similarly for $D f_{p}$ at any $u \in \Sigma_{p}$.

However, a $K$-affine function (more generally, a continuous " $\lambda$-concave" function) on a space with curvature bounded below is locally Lipschitz on the interior, as follows from the theory of quasigeodesics [Petrunin $\geq 2008$; Perelman and Petrunin 1994]. Indeed, locally in the interior, geodesics can be extended as quasigeodesics for a fixed distance, and the $\lambda$-concavity inequality $(f \circ \gamma)^{\prime \prime} \leq-2 \lambda$ holds on the extensions [Perelman and Petrunin 1994, 5.1, 6.1]. Similarly, a $K$-affine function on a locally compact, geodesically complete space of curvature bounded above is 
locally Lipschitz. For any locally Lipschitz $K$-affine $f$ on a space of curvature bounded below or above, $D f_{p}$ is well-defined and Lipschitz (see [Kleiner 1999]), and is a 1-affine function on a space with curvature bound 1 (see Section 4). In particular, condition (a) always holds in the interior of $X$ if $f$ is $K$-affine (in fact $f \in D^{r}$, with $1 \leq r \leq \operatorname{dim} X$ ), and we shall show that the same is true for condition (b). At points of $\partial X$, we have chosen the more geometric condition $(\dagger)$.

An analogue of Theorem 2.1 holds in the CBA setting if we assume local compactness and geodesic completeness (meaning that every geodesic can be extended to $\mathbb{R}$ ). In addition to the spaces of type (1)-(5) listed in Theorem 2.1, we find that $K$-affine functions for $K \neq 0$ can also be supported by spaces constructed by gluing together those of type (1) and (2). For example, consider a necklace of $m$ copies of $S^{n}$, each glued at its north and south poles to the adjacent ones. If $m$ is even, this space carries an 1-affine function whose restriction to each copy of $S^{n}$ is the cosine of distance from one of the poles.

Theorem 2.2. Let $X$ be a complete, locally compact, geodesically complete Alexandrov space of $C B A$ by $\bar{K}$. Then $X$ carries a nonconstant $K$-affine function $f$ if and only if $\bar{K} \geq K$ and $X$ splits as a cone over a complete, geodesically complete Alexandrov space $F$, in one of the following ways:

(1) $K>0$ and $X$ is obtained from a family of spherical suspensions

$$
X_{\alpha}=[0, \pi / \sqrt{K}] \times{\operatorname{sn}_{K} x}_{\alpha},
$$

where the $F_{\alpha}$ are CAT(1) spaces, by gluing a pole of $X_{\alpha_{1}}$ to a pole of $X_{\alpha_{2}}$, with $\alpha_{2} \neq \alpha_{1}$, so that any loop in the gluing pattern contains an even number of $X_{\alpha}$ 's (the "even loop condition").

(2) $K<0$ and $X=[0, \infty) \times_{\operatorname{sn}_{K} x} F$ is an elliptic cone over a (not necessarily connected) $C A T(1)$ space.

(3) $K<0$ and $X=\mathbb{R} \times{ }_{\exp \sqrt{-K} x} F$, a parabolic cone over an Alexandrov space $F$ of $C B A$ by 0 .

(4) $K<0$ and $X=\mathbb{R} \times{ }_{\cosh \sqrt{-K} x} F$, a hyperbolic cone over an Alexandrov space $F$ of $C B A$ by $\bar{K}$.

(5) $K=0$ and $X=\mathbb{R} \times F$, a Cartesian product, where $F$ is an Alexandrov space of $C B A$ by $\bar{K}$.

In cases (1), (2) and (3), X has CBA by $K$.

In cases (1) and (2) of Theorems 2.1 and 2.2, the fiber $F$ is the direction space at a critical point of $f$. If $X$ is Riemannian, this means $F=S^{n-1}$. The following Riemannian rigidity theorem is a corollary. For $K>0$, it is due to Obata [1962] (the only reference we know that considers $K$-affine functions for $K \neq 0$ ), and for $K=0$, to Innami [1982]. We shall see that these two Riemannian rigidity theorems have a companion for $K<0$ : 
Corollary 2.3. Suppose $X$ is a complete Riemannian manifold without boundary, carrying a nonconstant $K$-affine function. Then $X$ is isometric to $S^{n}(K)$ if $K>0$, to a Cartesian product with a line if $K=0$, and to $H^{n}(K)$ if $K<0$ and $f$ has a critical point.

\section{Structure of spaces carrying $\boldsymbol{K}$-affine functions}

Now we give more specific information on the "only if" implication in Theorems 2.1 and 2.2. A critical point of $f$ is a point $p$ such that $D f_{p}=0$. A gradient vector of $f$ at $p$ is an element $\nabla f_{p}$ of the tangent cone $C_{p}=[0, \infty) \times_{x} \Sigma_{p}$ whose direction $u \in \Sigma_{p}$ is a maximum point of $D f_{p}$ and whose length is $\left|\nabla f_{p}\right|=D f_{p}(u)$. We say $u$ is a gradient direction, and denote it by $\hat{\nabla} f_{p}$.

Theorem 3.1. Let $X$ be a complete, finite-dimensional Alexandrov space with curvature bounded below by $\bar{K}$. If $X$ carries a nonconstant $K$-affine function $f$ that satisfies the boundary condition $(\dagger)$, then $\bar{K} \leq K$ and:

(a) If $f$ has a critical point $p$, either:

(1) $K>0, f=\lambda \operatorname{cs}_{K} d_{p}$, and $X=[0, \pi / \sqrt{K}] \times_{\operatorname{sn}_{K} x} F$, a spherical suspension of the direction space $F=\Sigma_{p}$.

(2) $K<0, f=\lambda \operatorname{cs}_{K} d_{p}$, and $X=[0, \infty) \times_{\operatorname{sn}_{K} x} F$, an elliptic cone over the direction space $F=\Sigma_{p}$.

(b) If $f$ has no critical point, either:

$(3,4) K<0, \quad|\nabla f|^{2}+K f^{2}=E$ for some constant $E \geq 0$, and $X=\mathbb{R} \times{ }_{g} F$, a hyperbolic or parabolic cone with warping function $g \in \mathscr{F} K$, where $g \circ \pi_{\mathbb{R}}=|\nabla f|$ and the fiber $F$ has curvature $\geq \bar{K} E$. If $E=0$, we have $f=\exp \sqrt{-K} d_{Z}$, where $Z=f^{-1}(1)$. If $E>0$, we have

$$
f=\sqrt{-E / K} \sinh \sqrt{-K} d_{Z},
$$

where $Z=f^{-1}(0)$.

(5) $K=0, f=\lambda d_{Z}$, where $Z=f^{-1}(0)$ and $d_{Z}$ is signed distance to $Z$, and $X=\mathbb{R} \times F$, a Cartesian product with the factor $F$ isometric to $Z$.

Theorem 3.2. Let $X$ be a complete, locally compact, geodesically complete Alexandrov space with curvature bounded above by $\bar{K}$. If $X$ carries a nonconstant $K$ affine function $f$, then $\bar{K} \geq K$ and the conclusions of Theorem 3.1 hold, but with gluings satisfying the even loop condition allowed in (1), and “ $\leq \bar{K} E$ ” instead of “ $\geq \bar{K} E$ ” in $(3,4)$.

The proof will be in the following steps. Steps (i) and (ii) for the CBB case will be similar to arguments in [Mashiko 1999; 2002; Perelman and Petrunin 1994], but the rest of the proof uses a new method. A gradient geodesic of $f$ is a geodesic with right-hand tangents everywhere equal to a gradient direction of $f$. 
(i) At any noncritical point $p \in X$, the gradient directions $\hat{\nabla}( \pm f)_{p}$ are unique, and the direction space $\Sigma_{p}$ is a spherical suspension of $\left\{v \in \Sigma_{p}: D f_{p}(v)=0\right\}$ with $\hat{\nabla}( \pm f)_{p}$ as poles.

(ii) Let $p$ be a noncritical point of $f$ and let $A$ be the level set $f^{-1}(r)$ for $f(p)<$ $r<\sup f$. Then there is a unique minimizer from $p$ to $A$, and it is the unique gradient geodesic from $p$ to $A$.

(iii) The level sets of $f$ are equidistant from one another, $|\nabla f|$ is constant on each level set, and $|\nabla f|^{2}+K f^{2}$ is constant on $X$.

(iv) The level sets of $f$ are homothetic.

\section{Background}

A $C A T(K)$ space is a metric space in which any two points at distance less than $\pi / \sqrt{K}(=\infty$ if $K \leq 0)$ are joined by a distance-realizing geodesic, and any triangle $\triangle$ of perimeter less than $2 \pi / \sqrt{K}$ is thinner than its model triangle in the simply connected, 2-dimensional space form $S_{K}$ of curvature $K$. That is, the distance between any two points of $\triangle$ is no greater than the distance between the corresponding points on a triangle with the same side lengths in $S_{K}$. We assume the space is intrinsic, but allow infinite distance between points not joined by a rectifiable path. A metric space has $C B A$ by $K$ if it is locally CAT $(K)$. For CBB by $K$, inequalities are reversed. An Alexandrov space of $C B B$ by $K$ is a complete, locally compact length space satisfying triangle comparisons for triangles in some neighborhood of each point. Here, we assume connectedness, but also allow the exceptional case of two points. We shall only consider such spaces of finite dimension [Burago et al. 1992; 2001]. There is no distinction between the local and global triangle comparison properties in complete CBB spaces [Burago et al. 1992], or in complete, simply connected spaces with CBA by $K \leq 0$ ([Gromov 1981; Alexander and Bishop 1998]; see also [Ballmann 1995; Bridson and Haefliger 1999]). (In the case of CBB by $K>0$, circles of length $>2 \pi / \sqrt{K}$ and intervals of length $>\pi / \sqrt{K}$ are understood to be excluded.) Recent texts and monographs on Alexandrov spaces include [Ballmann 1995; Berestovskii and Nikolaev 1993; Bridson and Haefliger 1999; Burago et al. 2001; Buyalo 1995; Jost 1997; Plaut 2002]; see also [Gromov 1999, $\left.\$ 1.19_{+}\right]$.

The direction space $\Sigma_{p}$ at a point $p$ has CBB by 1 in a CBB space [Burago et al. 1992], and is CAT(1) in a CBA space [Nikolaev 1995]. If $f$ is Lipschitz and $K$-affine, the differential $D f_{p}$ is well defined and 1-affine on $\Sigma_{p}$, and its extension to a homogeneous function of degree 1 on the tangent cone $C_{p}=[0, \infty) \times_{x} \Sigma_{p}$ is affine. Indeed, both $f$ and $-f$ are $\mathscr{F} K$-convex, and so $D f_{p}$ and $-D f_{p}$ inherit $\mathscr{F} 1$-convexity on $\Sigma_{p}$, as is discussed in [Alexander and Bishop 2003].

One property not inherited by the tangent cone in the general case of CBA is geodesic completeness; that is why we have assumed local compactness. The following inheritance property is used repeatedly to get briefer proofs in the CBA 
case, and we do not know alternative proofs of all parts in the non locally compact case.

Theorem 4.1 [Buyalo 1995, Theorem 6.4]. If a complete, locally compact CBA space is geodesically complete, so are its direction spaces and tangent cones. Every direction is represented by a geodesic.

\section{Proof of Theorems 3.1 and 3.2}

Throughout this section, $f$ is a nonconstant $K$-affine function on $X$, where $X$ is either a complete CBB space with empty boundary (see Remark 5.5) or a complete, locally compact, geodesically complete CBA space. Then $f$ is locally Lipschitz (see Section 2). We carry out the steps listed in Section 3, beginning with a lemma on maximum points.

Lemma 5.1. If $p$ is a local maximum point of $f$, then $p$ is a critical point of $f$, $K \neq 0$, and $f=f(p) \operatorname{cs}_{K} d_{p}$.

Proof. For the CBB case the essential part of the proof has been given in [Perelman and Petrunin 1994, Lemma 6.2]. It proceeds by induction on dimension. For $\operatorname{dim} X=1$ the space consists of a single geodesic; clearly $f=f(p) \operatorname{cs}_{K} d_{p}$. If the result is true for dimensions $<n=\operatorname{dim} X$, then we can apply it to $D f_{p}$ on $\Sigma_{p}$ at a maximum point $u$. Thus we conclude that $D f_{p}=D f_{p}(u) \operatorname{cs}_{K} d_{p}$, and since $p$ is a maximum point, $D f_{p} \leq 0$. Hence $D f_{p}(u)=0$ and then $D f_{p}=0$. Since $f$ is $K$-affine, the initial conditions along any geodesic starting at $p$ determine $f$ and are simply that $f(p)$ is the initial value and $D f_{p}=0$ is the initial derivative. Hence $f=f(p) \operatorname{cs}_{K} d_{p} . K \neq 0$ by the assumption that $f$ is nonconstant.

For the CBA case we again have $D f_{p} \leq 0$. But for all $u \in \Sigma_{p}$, a geodesic extension determines an opposite direction $v$ for which $D f_{p}(v)=-D f_{p}(u) \leq 0$, so $D f_{p}(u)=0$. The formula for $f$ follows from the initial conditions as before.

Proof of Step $(i), C B B$. Suppose $p$ is a noncritical point of $f$. Since the function $h=D f_{p}$ on the compact space $\Sigma_{p}$ is 1 -affine and nonzero, it has a maximum point $u$, and $h=h(u) \cos d_{u}$ by Lemma 5.1. For $-h$ there is also a maximum point $v$, which must be at distance $\pi$ from $u$, since the formula yields critical points only at multiples of $\pi$ and the diameter of $\Sigma$ is at most $\pi$. Moreover, since $u$ and $v$ realize the maximum possible diameter, $\Sigma$ is the spherical suspension of their midpoint set, $h^{-1}(0)$.

Proof of Step (i), CBA, topological structure. The existence of maximum and minimum points for $h=D f_{p}$ again follows from the compactness of $\Sigma_{p}$. By Lemma 5.1, local extrema are isolated critical points of $h$, at least $\pi$ apart, with the local maximum values the same, nonzero and the negative of the local minimum values. Then there is a unique maximum (and minimum) point, since if there were two, representative geodesic segments beginning at $p$ could be joined to form a longer geodesic segment with those two directions opposite at $p$. 
Now let $u, v$ be the maximum and minimum points. For any $z \in h^{-1}(0)$ there is a unique minimizer of length $\pi / 2$ connecting $z$ to $u$, and similarly for $v$. These minimizers vary continuously with $z$, and for every other direction $w$ the unique minimizer to the closer of $u, v$ can be extended to reach $h^{-1}(0)$. This establishes the topological suspension structure of $\Sigma_{p}$ over $h^{-1}(0)$. We delay establishing the metric structure until after the next step.

Proof of Step (ii). Let $p$ be a noncritical point of $f$, and $A$ be the level set $f^{-1}(r)$ for $f(p)<r<\sup f$. First we show there is a minimizer from $p$ to $A$, and any such is a gradient geodesic. If $f$ has a maximum point $q$, then $f=f(q) \operatorname{cs}_{K} d_{q}$ and a minimizer $\gamma$ from $p$ to $q$ crosses $A$. It is a gradient geodesic of $f$ up to $q$, since the tangent directions to any level set of $d_{q}$ make an angle of $\pi / 2$ with the minimizers from $q$. Those tangent directions constitute exactly the zero set of $D f_{p}$, and as we just showed in the preceding step, the directions at angle $\pi / 2$ are $\hat{\nabla}( \pm f)_{p}$.

If sup $f$ is not realized, $A$ is nonempty, because for any $q$ such that $r<f(q)<$ sup $f$, a minimizer from $p$ to $q$ must cross $A$. Let $\gamma$ be a minimizer from $p$ to $A$. Then $\gamma^{\prime}(0)$ maximizes $D f_{p}$ among directions in $\Sigma_{p}$ that are tangent to geodesics to $A$. This is because any other geodesic from $p$ to its first intersection with $A$ is at least as long as $\gamma$; along such a geodesic, $f$ is a strictly increasing element of $\mathscr{F} K$, uniquely determined by its two end values and the length, and having initial derivative no more than that for $\gamma$. Thus, to show that $\gamma$ is a gradient geodesic, it suffices to show that max $D f_{\gamma(t)}$ is realized by a direction tangent to a geodesic extending to $A$. This is certainly true for CBA. For CBB, it can be obtained using the properties of quasigeodesics developed in [Perelman and Petrunin 1994]; but a more elementary argument is not long, so we give it now.

We may take $\gamma(t)=p$, and construct a sequence of geodesics $\gamma_{i}$ from $p$ to $A$, with initial directions converging to $\hat{\nabla} f_{p}$. The end in $A$ of each of these geodesics is the end of an approximate gradient curve $\sigma_{i}$ constructed by transfinite induction. We obtain $\sigma_{i}$ as a chain of geodesic segments and limit points defined for each ordinal. Specifically, start with $p_{1}=p$; for a successor ordinal $\alpha+1$, attach a geodesic segment, starting at $p_{\alpha}$ and ending at $p_{\alpha+1}$ for which $f\left(p_{\alpha+1}\right) \leq r$, with initial direction at distance less than $1 / i$ from $\hat{\nabla} f_{p_{\alpha}}$; for a limit ordinal $\alpha$ let $p_{\alpha}=\lim _{\beta<\alpha} p_{\beta}$. The construction needs a few remarks for justification. There is a geodesic $\tau$ on which $f$ increases, which starts at $p$ and ends after crossing $A$. Suppose the initial direction of $\tau$ is not $\hat{\nabla} f_{p}$. Then the rate of increase of $f$ along $\sigma_{i}$, for $i$ sufficiently large, will be greater than on $\tau$ at points with the same arclengths from $p$. Thus the points $p_{\beta}$ in the definition of $p_{\alpha}$ for a limit ordinal $\alpha$ will have Cauchy subsequences whose limit is $p_{\alpha}$. Moreover, the 2-point determination of $f \circ \gamma_{i}$ is based on an even shorter curve than $\sigma_{i}$ for the amount of change in $f$, so the initial derivative of $f$ must be greater than for $\sigma_{i}$. Therefore, $\lim _{i \rightarrow \infty} \sigma_{i}^{\prime}(0)=\hat{\nabla} f_{p}$. 
Then a convergent subsequence of $\sigma_{i}$ will have a limit geodesic $\gamma$ tangent to $\hat{\nabla} f_{p}$, and $\gamma$ will be no longer than any other geodesic from $p$ to $A$.

It remains to show the uniqueness of gradient geodesics. We have already proved the uniqueness of the direction at a point. For $\mathrm{CBB}$, uniqueness is immediate because geodesics cannot bifurcate. For CBA, it is certainly possible, even in a space carrying a $K$-affine function, for geodesics to bifurcate. Examples are given by applying Theorem 6.1 to a fiber $F$ with geodesic bifurcations. However, there can be no bifurcating pair of gradient geodesics of a $K$-affine function. This follows from Lemma 5.2 below, according to which the distance between gradient geodesics connecting two level sets is governed by a first-order differential equation with Lipschitz defining function having 0 as a solution.

Completion of proof of Step (i), CBA. We must show that the metric stucture of $\Sigma_{p}$ is that of a spherical suspension, or equivalently, that any two points of the cone $C_{p}$ lie in a flat strip. Since $D f_{p}$ is a nonzero affine function on $C_{p}$, the level sets are totally convex and the nearest point projection to a level set is nonexpanding. As we have just seen, this means that gradient geodesics extend to all of $\mathbb{R}$ and have bounded separation in both directions. Thus the Flat Strip Theorem applies [Bridson and Haefliger 1999, p. 182].

The remaining steps will depend on the following lemma, as did the uniqueness of gradient geodesics in the CBA case of Step (ii). The lemma is local, but we shall not lengthen the statement with local specifications, except to note that the distances $w(t)$ must be sufficiently small when $K>0$ that $\operatorname{sn}_{K} w(t)$ is never 0 .

Lemma 5.2. Let $A^{\prime}=f^{-1}\left(a^{\prime}\right)$ and $A=f^{-1}(a)$ be level sets for which no critical value of $f$ is between $a^{\prime}$ and $a$. Let $\gamma_{-}, \gamma_{+}$be minimizers from $p_{-}, p_{+} \in A^{\prime}$ to $A$, parametrized by the values $t$ of $f$, and denote by $E_{-}, E_{+}$the constant values of $|\nabla f|^{2}+K f^{2}$ along $\gamma_{-}, \gamma_{+}$. Then the distance $2 w=2 w(t)=d\left(\gamma_{-}(t), \gamma_{+}(t)\right)$ satisfies the differential equation

$$
2 \frac{d w}{d t}=-\frac{t K \operatorname{sn}_{K} w}{\operatorname{cs}_{K} w}\left(\frac{1}{E_{-}-K t^{2}}+\frac{1}{E_{+}-K t^{2}}\right) .
$$

Proof. By Step (ii), $\gamma_{-}$and $\gamma_{+}$are reparametrized gradient geodesics (uniqueness is not assumed). We use first variation for $2 w$, which requires us to prove that various limits of minimizers connecting $\gamma_{-}(t)$ and $\gamma_{+}(t)$ make the same angles with $\gamma_{-}$and $\gamma_{+}$[Burago et al. 2001]. Along a minimizer $\rho_{t}$ between $\gamma_{-}(t), \gamma_{+}(t)$ parametrized by arc length, $f \circ \rho_{t}(u)=m_{t} \operatorname{cs}_{K}(u),-w(t) \leq u \leq w(t)$. Let $\theta_{-}$be the angle between $\rho_{t}$ and $\gamma_{-}$, and $\theta_{+}$be the angle between oppositely directed $\rho_{t}$ and $\gamma_{+}$. Then

$$
\begin{aligned}
D f_{\gamma_{-}(t)}\left(\rho_{t}^{\prime}(-w(t))\right) & =\left|\nabla f_{\gamma_{-}(t)}\right| \cos \theta_{-}=m_{t} \operatorname{cs}_{K}^{\prime}(-w(t)) \\
& =-m_{t} K \operatorname{sn}_{K}(-w(t))=m_{t} K \operatorname{sn}_{K}(w(t)),
\end{aligned}
$$


and $\left|\nabla f_{\gamma_{+}(t)}\right| \cos \theta_{+}$is the same, by the symmetry of $f \circ \rho_{t}$. Thus $\cos \theta_{ \pm}$does not depend on the choice of minimizer $\rho_{t}$. Evaluating $f$ at an end of $\rho_{t}$ gives $t=m_{t} \operatorname{cs}_{K}(w(t)), m_{t}=t / \operatorname{cs}_{K}(w(t))$.

By first variation,

$$
\begin{aligned}
2 \frac{d w}{d t} & =-\left(\cos \theta_{-} \frac{d s_{-}}{d t}+\cos \theta_{+} \frac{d s_{+}}{d t}\right)=-\cos \theta_{-}\left|\nabla f_{\gamma_{-}}\right|^{-1}-\cos \theta_{+}\left|\nabla f_{\gamma_{+}}\right|^{-1} \\
& =-m_{t} K\left(\operatorname{sn}_{K} w\right)\left|\nabla f_{\gamma_{-}}\right|^{-2}-m_{t} K\left(\operatorname{sn}_{K} w\right)\left|\nabla f_{\gamma_{+}}\right|^{-2}
\end{aligned}
$$

from which (5-1) follows.

In order to apply the differential equation (5-1), we need two lemmas:

Lemma 5.3. The gradient-length function $|\nabla f|$ is locally Lipschitz.

Proof. This is clear if $f$ has a critical point $p$, since then $|\nabla f|=\left|f(p) K \operatorname{sn}_{K} d_{p}\right|$ and $d_{p}$ is Lipschitz. Otherwise, we restrict to an open set on which $f$ has Lipschitz constant $C$. It has been shown for Step (ii) that for a sufficiently small closed ball $\bar{B}_{r}(p)$, the gradient geodesic from the center $p$ is the minimizer to the maximum point $q$ of $f$ on the ball and $q$ is on the boundary of the ball. Define $F(p, r)=f(q)$. Then $F(p, r+s)=f\left(q^{\prime}\right)$, where $d\left(q, q^{\prime}\right)=s$; hence

$$
F(p, r) \leq F(p, r+s)=f(q)+f\left(q^{\prime}\right)-f(q) \leq F(p, r)+C s .
$$

Then $\bar{B}_{r}(p) \subset \bar{B}_{r+s}\left(p^{\prime}\right)$, where $s=d\left(p, p^{\prime}\right)$, so

$$
F(p, r)=f(q) \leq F\left(p^{\prime}, r+s\right)=f\left(q^{\prime \prime}\right) \leq F\left(p^{\prime}, r\right)+C d\left(p, p^{\prime}\right),
$$

where $q^{\prime \prime} \in \bar{B}_{r+s}\left(p^{\prime}\right)$. That is, $F(p, r)$ is Lipschitz continuous in both variables.

Along gradient geodesics, $f$ satisfies $f=f(p) \operatorname{cs}_{K}(s)+|\nabla f|(p) \operatorname{sn}_{K}(s)$, where $s$ is arc length. Then $f(q)=f(p) \operatorname{cs}_{K} r+|\nabla f|(p) \operatorname{sn}_{K}(r)$, so

$$
|\nabla f|(p)=\left(f(q)-f(p) \operatorname{cs}_{K} r\right)\left(\operatorname{sn}_{K} r\right)^{-1}=\left(F(p, r)-f(p) \operatorname{cs}_{K} r\right)\left(\operatorname{sn}_{K} r\right)^{-1} .
$$

Hence,

$$
\begin{aligned}
|| \nabla f|(p)-| \nabla f\left|\left(p^{\prime}\right)\right| & =\left|F(p, r)-f(p) \operatorname{cs}_{K} r-F\left(p^{\prime}, r\right)+f\left(p^{\prime}\right) \operatorname{cs}_{K} r\right|\left(\operatorname{sn}_{K} r\right)^{-1} \\
& \leq C\left(1+\operatorname{cs}_{K} r\right)\left(\operatorname{sn}_{K} r\right)^{-1} d\left(p, p^{\prime}\right) .
\end{aligned}
$$

Lemma 5.4. If $A=f^{-1}(a)$ is a noncritical level set, the nearest point map from a neighborhood of A to A is locally Lipschitz.

Proof. By Lemma 5.2, the distances $2 w\left(a^{\prime}\right)$ between pairs of points on a nearby level set $f^{-1}\left(a^{\prime}\right)$ are initial values for the differential equation (5-1) and the extrinsic distances in $A$ are the final values $2 w(a)$. The right-hand side of (5-1) depends on the parameters $E_{-}=\left|\nabla f_{p_{-}}\right|^{2}+K f\left(p_{-}\right)^{2}$ and $E_{+}=\left|\nabla f_{p_{+}}\right|^{2}+K f\left(p_{+}\right)^{2}$. By Lemma 5.3 and the assumption on $f$, this dependence is locally Lipschitz. Thus $2 w(a)$ must also be a locally Lipschitz function of $p_{ \pm}$, and hence also the nearestpoint map between level sets. 
More generally, the local Lipschitz property of the nearest point map from a whole neighborhood of $A$ to $A$ follows from that for pairs of points on the same level set, using the triangle inequality. Indeed, for $p_{-}, p_{+}$in a neighborhood of $A$, let $p_{-}^{\prime}$ be the nearest point to $p_{-}$on the level set through $p_{+}$. Then $d\left(p_{-}^{\prime}, p_{+}\right) \leq d\left(p_{-}^{\prime}, p_{-}\right)+d\left(p_{-}, p_{+}\right) \leq 2 d\left(p_{-}, p_{+}\right)$, so that the Lipschitz constant for the projection from a neighborhood is at most twice that for the projection from level sets.

Now we are ready to complete steps (iii) and (iv), and Theorems 3.1 and 3.2. Comparing with Mashiko's proof [2002] for CBB by 0, we note that the analogue there of Step (iii) is a global argument that fails for CBB by $K<0$. Another concern here is the intrinsic geometry of level sets; since they are not totally geodesic when $K \neq 0$, we must consider how to transmit a precise curvature bound from a warped product to its fiber.

Proof of Step (iii). By Lemma 5.4, any pair $p_{ \pm} \in A^{\prime}$ is joined by a rectifiable curve in $A^{\prime}$, since the projection to $A^{\prime}$ of any rectifiable curve connecting them in a neighborhood of $A^{\prime}$ is again rectifiable. For such a join a direction exists almost everywhere. Those directions and their images under the nearest point map to $A$ are perpendicular to the gradient directions of $f$, so that by first variation the gradient geodesics have constant length as their initial points move along the curve in $A^{\prime}$. This proves equidistance, from which the other claims follow.

Proof of Step (iv). By Step (iii) the constants $E_{ \pm}$in the differential equation of Lemma 5.2 for $2 w(t)$ are the same and independent of $p_{ \pm}$. Thus the differential equation gives a constant ratio between the "input" initial value $w\left(a^{\prime}\right)$ and the "output" final value $w(a)$ which depends only on $a^{\prime}, a$ and that initial value. Then $\lim w(a) / w\left(a^{\prime}\right)$ as $w\left(a^{\prime}\right) \rightarrow 0$ is the magnification factor on intrinsic distances of the nearest-point map from $A^{\prime}$ to $A$.

Proof of Theorems 3.1 and 3.2. We must identify the magnification factor of Step (iv) in terms of $f$ and choose a level set of $f$, appropriately scaled, to be the fiber $F$ of a warped product. To identify the magnification factor we again refer to the differential equation of Lemma 5.2, writing $E_{ \pm}=E=|\nabla f|^{2}+K f^{2}$. It is also more convenient to regard $w=w(t, v)$, with $v=w\left(a^{\prime}\right)$ as the initial value, as an analytic function of two variables (since the defining function is analytic). Then the magnification factor at $t$ is $z(t)=(\partial w / \partial v)(t, 0)=\lim _{v \rightarrow 0}(w(t, v) / v)$, since $w(t, 0)=0$. Thus $d z / d t=\left(\partial^{2} w / \partial t \partial v\right)(t, 0)=\left(\partial^{2} w / \partial v \partial t\right)(t, 0)=\lim _{v \rightarrow 0}\left(\frac{\partial w}{\partial t} / v\right)$. Substituting (5-1) gives

$$
d z / d t=-K t z\left(E-K t^{2}\right)^{-1} .
$$

This is easily solved by separation of variables:

$$
z=\sqrt{E-K t^{2}} / \sqrt{E-K\left(a^{\prime}\right)^{2}}=|\nabla f|_{f=t} /|\nabla f|_{f=a^{\prime}} .
$$


It follows that the closure of each component of $X-$ \{critical points $\}$ is a warped product, $X_{\alpha}=I \times{ }_{g_{\alpha}} F_{\alpha}$, and the lift of $g_{\alpha}$ to $X_{\alpha}$ is a constant multiple of $|\nabla f|$. $g \in \mathscr{F} K$ implies $X_{\alpha}$ contains totally geodesic surfaces isometric to domains in $S_{K}$ (namely, those projecting to geodesics of the fiber). Therefore $\bar{K} \leq K$ in Theorem 3.1 (CBB), and $\bar{K} \geq K$ in Theorem 3.2 (CBA).

Cases (1) and (2): By Lemma 5.1, $|\nabla f|$ is $\left|f\left(q_{\alpha}\right) K \mathrm{sn}_{K} d_{q_{\alpha}}\right|$, where $q_{\alpha}$ is a critical point, and we may scale $F$ so that $X_{\alpha}=[0, \pi / \sqrt{K}] \times{ }_{\mathrm{sn}_{K} x} F_{\alpha}$. The tangent cone $C_{\alpha}=[0, \infty) \times_{x} \Sigma_{\alpha}$ to $X_{\alpha}$ at $q_{\alpha}$ is the blowup of $X_{\alpha}$ at $q_{\alpha}$. Since $\operatorname{sn}_{K}^{\prime}(0)=1$, it follows that $C_{\alpha}=[0, \infty) \times_{x} F_{\alpha}$, hence $\Sigma_{\alpha}=F_{\alpha}$.

For CBB, there is only one component $X=X_{\alpha}$, because geodesics cannot bifurcate. For CBA, any collection of connected CAT(1) spaces $\Sigma_{\alpha}$ may occur, and this describes all possible $X$ 's in CBA case (2). In CBA case (1), i.e., when $K>0$, more $X_{\alpha}$ 's may be introduced subject to further gluings at the poles, subject only to the condition that there is a globally defined $K$-affine function $f$ whose restriction to each $X_{\alpha}$ is $f(q) \operatorname{cs}_{K} d_{q}$, where $q$ is a critical point. This simply means that maximum points may be glued to each other and minimum points may be glued to each other, but not maximum to minimum; equivalently, the even loop condition holds.

Cases $(3,4)$ : Along a gradient geodesic we may take $f^{\prime}=g$, and hence $g^{\prime}=$ $-K f$. If $E=0$, then $f=\exp \sqrt{-K} d_{Z}$, where $Z=f^{-1}(1)$. If $E>0$, then $f=\lambda \sinh \sqrt{-K} d_{Z}$, where $Z=f^{-1}(0), d_{Z}$ is signed distance to $Z$, and $-\lambda^{2} K=E$.

It remains to show that the fiber $F$ has curvature bound $\bar{K} E$. If $E>0$, then $g=\sqrt{E} \cosh \sqrt{-K} x$. At the minimum point of $g$, the copy $g(0) F$ of the fiber is totally geodesic in $X$, by the definition of warped product, and so has curvature bound $\bar{K}$. Since $g(0)=\sqrt{E}$, the desired curvature bound follows.

If $E=0$, we prove $F$ has curvature bound 0 by showing that angular excesses of triangles in $F$ are nonnegative (CBB) or nonpositive (CBA). By scaling of the metric and $f$, we reduce to the case $K=-1, f=g=\exp$. First consider CBB by $\bar{K}$, so global triangle comparisons hold in $X$. For a triangle $T$ in $F$, the image in $X$ of each side $\tau$ sweeps out, as $x$ varies, a totally geodesic surface $S_{\tau}$, isometric to a convex region in the hyperbolic plane and in which each $\{x\} \times \tau$ is a horocyclic arc. The side lengths of the triangle $\{x\} \times T$ are scaled by $e^{x}$ relative to those of $T$, while the (upper) angles $\alpha, \beta, \gamma$ are the same. Consider a triangle $T_{x}$ in $X$ with the same vertices as $\{x\} \times T$, where each side of $T_{x}$ is a chord in $S_{\tau}$ of a horocyclic side $\tau$. Hence $T_{x}$ has angles $\alpha_{x}, \beta_{x}, \gamma_{x} \rightarrow \alpha, \beta, \gamma$ as $x \rightarrow-\infty$. But $\alpha_{x}+\beta_{x}+\gamma_{x} \geq-\bar{K} A_{x}+\pi$, where $A_{x}$ is the area of a model triangle for $T_{x}$, so by taking $x \rightarrow-\infty$ we obtain the desired comparison $\alpha+\beta+\gamma \geq \pi$.

For CBA the same proof works with the inequalities reversed, except that we must restrict to neighborhoods $U$ in $F$ such that all triangles $T_{x}$ with vertices in $\mathbb{R} \times{ }_{g} U$ admit comparisons. We show that such neighborhoods exist. For an open subset of a CBA space such that minimizers joining any pair of its points are unique 
and vary continuously, the corresponding triangles admit the Alexandrov patchwork construction, so they admit comparisons. Let $U$ be the projection of such an open set in $X$ to $F$. Then $U$ has the continuous bipoint uniqueness property: a minimizer projects to a minimizer, and if a pair in $U$ had a second minimizer in $F$, it could be combined with the projection to $\mathbb{R}$ of the original minimizer in $X$ to get a second minimizer in $X$ with the same ends. Continuity follows from local compactness and uniqueness. But then $\mathbb{R} \times{ }_{g} U$ has the bipoint uniqueness property since each $S_{\tau}$ does.

Remark 5.5. The assumption ( $\dagger$ ) in Theorem 3.1 allows us to assume $\partial X=\varnothing$. Indeed, by Perelman's doubling theorem [1991], the double $X^{*}$ of $X$ is a CBB space with empty boundary, and so our proof above applies to the tautological extension of $f$ to $X^{*}$. The uniqueness of gradient geodesics then implies that the splitting respects the doubling structure.

\section{Proof of Theorems 2.1 and 2.2}

Now we turn to the converse, which is all that remains to complete the proof of Theorems 2.1 and 2.2. When $K=0$, projection to the first factor of $\mathbb{R} \times F$ is affine. Let us construct $K$-affine functions on $K$-cones when $K \neq 0$. As was shown in [Alexander and Bishop 1998], in any warped product of geodesic metric spaces, the geodesics satisfy an energy equation, analogous to Clairaut's equation on a surface of revolution.

Theorem 6.1. Let $F$ be a geodesic metric space and $K \neq 0$. Let $g: I \rightarrow \mathbb{R}$ be a nonconstant function in $\mathscr{F} K$, where $I$ is the maximal interval containing 0 on which $g \geq 0$. Then there is a function $f \in \mathscr{F} K$ such that $f^{\prime}=g$, and whose lift to the warped product $X=I \times{ }_{g} F$ is $K$-affine.

Proof. Take $f=-K^{-1} g^{\prime}$, so $f^{\prime}=g$ and $f \in \mathscr{F} K$. From the energy equation we see that, letting $x$ be the standard coordinate on $I \subset \mathbb{R}$, the horizontal speed $v=|d x / d s|$ on any geodesic in $X$ with arclength parameter $s$ satisfies $v^{2}+\left(c^{2} / g^{2}\right)=1$ for some constant $c$ [Alexander and Bishop 1998]. Forgetting the distinction between $f$ and its lift $f \circ \pi_{I}$, write $d f / d s$ for the derivative of the latter along the geodesic. Then

$$
\begin{aligned}
\frac{d f}{d s} & =f^{\prime} \frac{d x}{d s}= \pm f^{\prime} v= \pm f^{\prime} \sqrt{1-\left(c^{2} / g^{2}\right)} \\
& =-\left( \pm g \sqrt{1-\left(c^{2} / g^{2}\right)}\right)=-\left( \pm \sqrt{g^{2}-c^{2}}\right),
\end{aligned}
$$

where \pm is the sign of $d x / d s$. Therefore

$$
\begin{aligned}
\frac{d^{2} f}{d s^{2}} & =-\left( \pm \frac{g(d g / d s)}{\sqrt{g^{2}-c^{2}}}\right) \\
& =-\left( \pm \frac{g g^{\prime}(d x / d s)}{\sqrt{g^{2}-c^{2}}}\right)=-\frac{g g^{\prime} \sqrt{1-\left(c^{2} / g^{2}\right)}}{\sqrt{g^{2}-c^{2}}}=-K f .
\end{aligned}
$$


Proof of Theorems 2.1 and 2.2. By the preceding theorem, the $K$-cones (1)-(5) in Theorems 2.1 and 2.2 carry $K$-affine functions. Since in the CBB case their construction respects doubling, $(\dagger)$ holds. It only remains to verify that $K$-cones have the stated curvature bounds. It is well-known that (1)-(3) have curvature bound $K$; see [Burago et al. 2001, pp. 132 and 355]. In (4), we are assuming that $K<0, g=\cosh \sqrt{-K} x$ and $F$ has curvature bound $\bar{K}$, where $\bar{K} \leq K$ for CBB and $\bar{K} \geq K$ for CBA; we claim that $X=\mathbb{R} \times{ }_{g} F$ has curvature bound $\bar{K}$. By the general criterion for curvature bounds for warped products [Alexander and Bishop $\geq 2008]$, we need only check for CBB that $g^{\prime \prime}+\bar{K} g \leq 0$ and that the curvature bound $\bar{K}$ of the fiber satisfies $\bar{K} \geq \bar{K} g^{2}$, and for CBA that $g^{\prime \prime}+\bar{K} g \geq 0$ and $\bar{K} \leq \bar{K}(\inf g)^{2}$. All of this is clear.

\section{Acknowledgments}

We thank Vitali Kapovitch and Anton Petrunin for helpful comments.

\section{References}

[Aleksandrov et al. 1986] A. D. Aleksandrov, V. N. Berestovskiǔ, and I. G. Nikolaev, "Generalized Riemannian spaces", Russian Math. Surveys 41:3 (1986), 1-54. MR 88e:53103 Zbl 0625.53059

[Alexander and Bishop 1998] S. B. Alexander and R. L. Bishop, "Warped products of Hadamard spaces”, Manuscripta Math. 96:4 (1998), 487-505. MR 99g:53070 Zbl 0909.53050

[Alexander and Bishop 2003] S. Alexander and R. L. Bishop, "F्F $K$-convex functions on metric spaces”, Manuscripta Math. 110:1 (2003), 115-133. MR 2004a:53100

[Alexander and Bishop $\geq 2008$ ] S. Alexander and R. L. Bishop, "Curvature bounds for warped products of metric spaces", http://www.math.uiuc.edu/ sba/wp.pdf. To appear in Geom. and Funct. Anal.

[Ballmann 1995] W. Ballmann, Lectures on spaces of nonpositive curvature, DMV Seminar 25, Birkhäuser, Basel, 1995. MR 97a:53053

[Berestovskii 1983] V. N. Berestovskiǔ, "Borsuk's problem on metrization of a polyhedron", Dokl. Akad. Nauk SSSR 268:2 (1983), 273-277. English translation: Soviet Math. Dokl. 27:1 (1983), 273-277. MR 85b:51017

[Berestovskii and Nikolaev 1993] V. N. Berestovskij and I. G. Nikolaev, "Multidimensional generalized Riemannian spaces", pp. 165-243, 245-250 in Geometry IV, edited by Y. G. Reshetnyak, Encyclopaedia Math. Sci. 70, Springer, Berlin, 1993. MR 1263965

[Bridson and Haefliger 1999] M. R. Bridson and A. Haefliger, Metric spaces of non-positive curvature, Grundlehren der Math. Wissenschaften 319, Springer, Berlin, 1999. MR 2000k:53038

[Burago et al. 1992] Y. Burago, M. Gromov, and G. Perelman, "A. D. Aleksandrov spaces with curvatures bounded below”, Uspekhi Mat. Nauk 47:2(284) (1992), 3-51, 222. English translation: Russian Math. Surveys 47:2 (1992), 1-58. MR 93m:53035

[Burago et al. 2001] D. Burago, Y. Burago, and S. Ivanov, A course in metric geometry, Graduate Studies in Math. 33, American Mathematical Society, Providence, RI, 2001. MR 2002e:53053 Zbl 0981.51016

[Buyalo 1995] S. Buyalo, "Lecture notes on spaces of nonpositive curvature", course taught at the University of Illinois Urbana-Champaign, 1995, http://www.pdmi.ras.ru/ sbuyalo/add.html. 
[Gromov 1981] M. Gromov, "Hyperbolic manifolds, groups and actions", pp. 183-213 in Riemann surfaces and related topics, edited by I. Kra and B. Maskit, Ann. of Math. Stud. 97, Princeton Univ. Press, Princeton, 1981. MR 82m:53035 Zbl 0467.53035

[Gromov 1999] M. Gromov, Metric structures for Riemannian and non-Riemannian spaces, Progress in Mathematics 152, Birkhäuser, Boston, 1999. MR 2000d:53065

[Innami 1982] N. Innami, "Splitting theorems of Riemannian manifolds", Compositio Math. 47:3 (1982), 237-247. MR 84e:53053 Zbl 0514.53040

[Jost 1997] J. Jost, Nonpositive curvature: geometric and analytic aspects, Lectures in Mathematics ETH Zürich, Birkhäuser Verlag, Basel, 1997. MR 98g:53070

[Kleiner 1999] B. Kleiner, "The local structure of length spaces with curvature bounded above", Math. Z. 231:3 (1999), 409-456. MR 2000m:53053

[Mashiko 1999] Y. Mashiko, “Affine functions on Alexandrov surfaces”, Osaka J. Math. 36:4 (1999), 853-859. MR 2001a:53057 Zbl 01455440

[Mashiko 2002] Y. Mashiko, "A splitting theorem for Alexandrov spaces", Pacific J. Math. 204:2 (2002), 445-458. MR 2003b:53044 Zbl 01819000

[Milka 1967] A. D. Milka, "Metric structure of a certain class of spaces that contain straight lines", Ukrain. Geometr. Sb. Vyp. 4 (1967), 43-48. MR 41 \#983

[Nikolaev 1995] I. Nikolaev, "The tangent cone of an Aleksandrov space of curvature $\leq K$ ", Manuscripta Math. 86:2 (1995), 137-147. MR 95m:53062 Zbl 0822.53043

[Obata 1962] M. Obata, "Certain conditions for a Riemannian manifold to be iosometric with a sphere”, J. Math. Soc. Japan 14 (1962), 333-340. MR 25 \#5479 Zbl 0115.39302

[Perelman 1991] G. Perelman, "Alexandrov's spaces with curvature bounded from below, II", preprint, 1991.

[Perelman and Petrunin 1994] G. Perelman and A. Petrunin, "Quasigeodesics and gradient curves in Alexandrov spaces”, preprint, 1994, http://www.math.psu.edu/petrunin/papers/qg_ams.pdf.

[Petrunin $\geq 2008$ A. Petrunin, in preparation.

[Plaut 2002] C. Plaut, "Metric spaces of curvature $\geq k$ ", pp. 819-898 in Handbook of geometric topology, North-Holland, Amsterdam, 2002. MR 2002m:53063

[Toponogov 1959] V. A. Toponogov, "Riemannian spaces containing straight lines", Dokl. Akad. Nauk SSSR 127 (1959), 977-979. English translation: "Spaces with straight lines", pp. 278-280 in Twenty-two papers on algebra, number theory and differential geometry, American Mathematical Society Translations 37, 1964. MR 21 \#7520 Zbl 0138.42902

Received February 13, 2004.

Stephanie B. Alexander

UNIVERSITY OF ILLINOIS AT URBANA-CHAMPAIGN

1409 W. GREEN ST.

URBANA, IL 61801

sba@math.uiuc.edu

RICHARD L. BISHOP

UNIVERSITY OF ILLINOIS AT URBANA-CHAMPAIGN

1409 W. GREEN ST.

URBANA, IL 61801

bishop@math.uiuc.edu 